\section{Diagnostic accuracy of ultrasound in suspected ovarian torsion}

\author{
Leena Mawaldi, ${ }^{1}$ Charu Gupta, ${ }^{1}$ \\ Hanadi Bakhsh, ${ }^{1}$ Maissa Saadeh, ${ }^{2}$ \\ Mostafa A. Abolfotouh ${ }^{2}$ \\ 'Department of Obstetrics and \\ Gynaecology, King AbdulAziz Medical \\ City, National Guard Health Affairs, \\ Riyadh; ${ }^{2}$ King Abdullah International \\ Medical Research Center (KAIMRC), King \\ Saud Bin-Abdulaziz University for Health \\ Sciences, National Guard Health Affairs, \\ Saudi Arabia
}

\section{Abstract}

Objective. Ultrasound has been proven to be useful in detecting underlying ovarian pathology. However, its role in the prediction of ovarian torsion has been controversial. The aim of the study was to assess the validity of ultrasound in the prediction of ovarian torsion in patients with acute pelvic pain related to clinically suspected ovarian torsion.

Materials and Methods. A retrospective observational study was conducted at the Obstetrics/Gyneacology department using a 10year chart review of all female patients older than 11 years of age with highly suspected ovarian torsion who underwent clinical assessment and ultrasound prior to surgery $(n=62)$. The sensitivity and specificity of ultrasound were determined by cross-tabulation of the ultrasound and surgical findings.

Results. Of the suspected cases, 54 (87.1\%) were confirmed to be cases of ovarian torsion by surgery. The majority of the cases were suggestive of ovarian torsion, which was indicated by clinical examination (77.4\%), ultrasound (77.4\%), or pathological examination (79\%). Almost one-half of the cases (46.8\%) showed a pain score $>6$; two-thirds (62.9\%) presented with vomiting and/or nausea; and more than one-third (38.7\%) presented with leukocytosis. The estimated sensitivity and specificity of ultrasound were 0.74 and 0.0 , respectively. The positive predictive value was 0.83 . Ultrasound was significantly associated with both clinical examination $(\mathrm{P}=0.039)$ and pain score $(\mathrm{P}=0.008)$.

Conclusion. The diagnosis of ovarian torsion cannot be exclusively based on ultrasound. Both clinical and sonographical evaluation of acute pelvic pain should be considered for the diagnosis. A definitive diagnosis remains challenging.

\section{Introduction}

Ovarian torsion refers to the twisting of the ovary on its ligamentous supports, which often results in an impedance of its blood supply. This condition is the fifth most common gynecological emergency and affects females of all ages. ${ }^{1}$ Expedient diagnosis is important to preserve ovarian function and prevent adverse sequelae, ${ }^{2}$ however, the diagnosis can be challenging because the symptoms are relatively nonspecific. The ovaries were examined in a large series of patients with surgically confirmed torsion-associated cysts in $48 \%$ and neoplasms in $46 \%$; the remainder occurred in normal-appearing ovaries. ${ }^{3}$ Histopathology was benign in over $90 \%$ of patients. ${ }^{3,4}$ Whereas anatomic factors usually account for ovarian torsion in adults, normal ovaries have been demonstrated in over $50 \%$ of ovarian torsion cases in children under the age of 15 years. ${ }^{5}$ In adults, torsion has also been described following laparoscopic hysterectomy, suggesting that even release of the fulcrum, on which the ovaries usually twist, does not protect against torsion. ${ }^{6}$ Strenuous exercise or a sudden increase in abdominal pressure also promotes torsion of the ovary around the vascular pedicle. ${ }^{7}$ The right ovary is more likely than the left to undergo torsion, suggesting that the sigmoid colon may help to prevent torsion. ${ }^{8}$

Women who are pregnant ${ }^{9,10}$ or are undergoing ovarian hyperstimulation during infertility treatment are at increased risk of ovarian torsion. ${ }^{11}$ The overall incidence of torsion in pregnant women was reported as $15 \% .^{12}$ In association with pregnancy, torsion most commonly occurred between 10 and 17 weeks of gestation and during the postpartum period. A much lower incidence was reported in another series of pregnant women. ${ }^{13,14}$ Torsion was half as common as appendicitis during pregnancy. ${ }^{15}$ The clinical presentation of ovarian torsion is nonspecific, and therefore, it is a challenge for the clinician to recognize this condition and differentiate it from other etiologies.

The two most common presenting features of ovarian torsion are acute pelvic pain (83\%) and an adnexal mass (72\%). ${ }^{16}$ Other symptoms and findings include nausea and vomiting (70 $\%)$, stabbing pain (70\%), sudden and sharp pain in the lower abdomen (59), pain radiating to the back, flank, or groin (51\%), peritoneal signs $(3 \%)$, fever $(<2 \%)$, leukocytosis, and an increased level of Interleukin-6. ${ }^{17,18}$

Ultrasound can detect adnexal lesions and ovarian enlargement. An enlarged, heterogeneous-appearing ovary is the most common ultrasound finding ${ }^{19}$ however, the presence of normal-appearing ovaries does not rule out the diagnosis..$^{20}$ Doppler ultrasound is also controversial, because it shows diminishing or absent ovarian vessel flow in two-dimensional
Correspondence: Prof. Mostafa A. Abolfotouh, Head of the Medical Team, Biobanking Section, King Abdullah International Medical Research Center, King Saud Bin-Abdulaziz University for Health Sciences, Riyadh 11426, POB 22490, Saudi Arabia.

Tel. +966.1.2520088x14469 - Fax: +966.1.2520772.

E-mail:mabolfotouh@gmail.com

Key words: acute pelvic pain, ultrasound, ovarian torsion.

Acknowledgements: the study was approved by the research committee of King Abdullah International Medical Research Center (KAIMRC), King Saud Bin-Abdulaziz University for Health Sciences. Special thanks go to Mr. Mahmoud Salam, the research coordinator at KAIMRC, for helping in reference formatting. The authors also thank the editing service office of KAIMRC for the English language editing of the manuscript via a specialist English language copyeditor (American Journal Experts).

Conflict of interest: the authors report no conflicts of interest.

Received for publication: 25 May 2011. Accepted for publication: 11 October 2011.

This work is licensed under a Creative Commons Attribution NonCommercial 3.0 License (CC BYNC 3.0).

(C) Copyright L. Mawaldi et al., 2011

Licensee PAGEPress, Italy

Urogynaecologia 2011; 25:e15

doi:10.4081/uij.2011.e15

color. $^{21}$ The aim of this study was to assess the validity of ultrasound in the prediction of ovarian torsion in patients with acute pelvic pain suggestive of ovarian torsion.

\section{Materials and Methods}

A retrospective observational study was conducted in the Department of Obstetrics and Gynaecology, King AbdulAziz Medical City, Riyadh, Saudi Arabia. All female patients $(n=62)$ older than 11 years of age who presented with acute pelvic pain with highly suspected ovarian torsion from January 2000 through December 2009 and for whom surgeries were performed within less than 6 hours of the ultrasound assessment were included. Patients underwent a transabdominal scan using 3-5 MHz probes and a 5-7 MHz endovaginal transducer with both real-time gray-scale and color Doppler imaging. The data were collected by chart review for all patients.

All categorical variables (age, marital status, pregnancy, pain score, vomiting, white blood 
cell counts, abdominal/pelvic exam, ultrasound, and surgery) were recorded, and their frequency distributions were measured.

Sensitivity, specificity, and predictive values were determined by cross-tabulation of the results of ultrasound and the surgical findings of ovarian torsion as the gold standard. Based on this tabulation, the sensitivity, specificity and positive predictive values were computed for ultrasound. The sensitivity of ultrasound diagnosis compared with the surgical diagnosis gold standard was determined by calculating how frequently the correct ultrasound diagnosis was made in each surgical diagnosis. The specificity of the ultrasound diagnosis was determined by calculating how frequently the ultrasound diagnosis was not made when the corresponding surgical diagnosis was not present. Positive predictability indicated how fre-

Table 1. Sociodemographic and clinical characteristics of the 62 cases of suspected ovarian torsion.

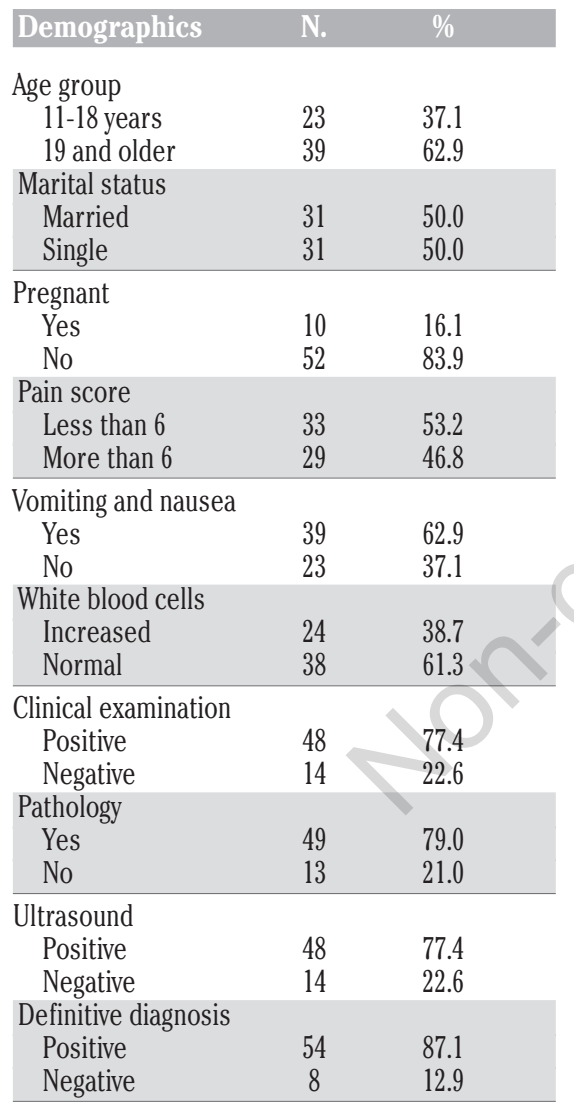

Table 2. $2 \times 2$ table of the ultrasound results and the definitive diagnosis of ovarian torsion.

\begin{tabular}{lccc} 
Ultrasound & Positive & Ovarian torsion & Total \\
Positive & 40 & 8 & 48 \\
Negative & 14 & 0 & 14 \\
\hline Total & 54 & 8 & 62 \\
\hline
\end{tabular}

Sensitivity, 40/54 = $74 \%$; Specificity, 0/8 = 0.0\%; positive predictive value (PPV), $40 / 45=83.3 \%$; negative predictive value (NPV), 0/14 = 0\%; Kappa, $0.20, P=0.102$ quently the ultrasound diagnosis correctly reflected the surgical diagnosis. In addition, the level of agreement between the ultrasound diagnosis and the surgical diagnosis was determined by calculating the kappa coefficient. The data were analyzed using SPSS version 15.0. All of the statistical tests were considered significant at $\mathrm{P}<0.05$.

\section{Results}

Table 1 shows a summary of the sociodemographic and clinical characteristics of the 62 cases of suspected ovarian torsion. Of these cases, 54 (87.1\%) were confirmed as cases of ovarian torsion by surgery. The majority of the cases were suggestive of ovarian torsion, as determined by clinical examination (77.4\%), ultrasound (77.4\%), or pathological examination (79\%). Almost one-half of the cases (46.8\%) were associated with a pain score $>6$; two-thirds (62.9\%) presented with vomiting and/or nausea; and more than one-third (38.7\%) presented with leukocytosis.

Table 2 shows that when ultrasound is used for predicting ovarian torsion, the sensitivity is $74 \%$. That is, the ultrasound scan correctly diagnosed $74 \%$ of ovarian torsion cases and missed $26 \%$ of these cases (false negatives). However, free subjects were misclassified as ovarian torsion cases (false positives).

Table 3 shows that ultrasound was significantly associated with clinical examination $(\mathrm{P}=0.039)$ and pain score $(\mathrm{P}=0.008)$.

Table 4 shows that positive ultrasound alone is not predictive of ovarian torsion (Positive predictive value, $P P V=0.0$ ). Additionally, examination and pain scores have 100\% PPV each, yet the yield is only $13 \%$ and $3.7 \%$, respectively. When relying upon ultrasound and one of these two techniques, the PPV reaches $87 \%$, and the yield is $74 \%$.

\section{Discussion}

Ovarian torsion is an uncommon condition; however, it is the most common gynecological surgical emergency and has an overall incidence of $2.7 \%{ }^{22}$ Awareness of its clinical and sonographical features may enable prompt treatment that can spare the ovary. The possible consequences of delayed diagnosis are serious, including ovarian necrosis, peritonitis, and death. Underlying pathology was found in $79 \%$ of the cases. Enlargement of the ovary was the common predisposing factor, including polycystic ovary, functional cyst, and ovarian hyperstimulation syndrome. There were cases of ovarian torsion without ovarian pathology due to an elongated utero-ovarian ligament in $21 \%$ of the cases.

Ultrasound has been proven to be useful in detecting any underlying ovarian pathology. Lee et al. concluded that identification of the twisted vascular pedicle through ultrasonography is suggestive of ovarian torsion, and color Doppler sonography could be helpful in predicting the viability of adnexal structures by depicting blood flow within the twisted vascular pedicle. ${ }^{21}$ Gray-scale findings typically include asymmetric enlargement, a solid heterogeneous appearance, and peripheral cystic areas; however, ultrasound was less capable of determining ovarian torsion. It shows a sensitivity of 0.74 (resulting in a false reassurance of $26 \%$ ), and a specificity of 0.0 (resulting in a false warning of 100\%), and a PPV of 0.83 (suggesting it might be useful in the clinical setting). Pena et al. concluded that abnormal flow detected by Doppler sonography is highly predictive of adnexal torsion and is therefore useful in the diagnosis of ovarian torsion; ${ }^{23}$ however, the detection of normal flow does not necessarily exclude ovarian torsion. Thus, our results are similar to those of international studies. The study by Cohen et al. concluded that patients and surgeons alike should be aware of the difficulty in making accurate preoperative diagnoses of acute gynecologic pathologies in the emergency room. ${ }^{24}$ In the present study, a pain score $>6(100.0)$ and an abdominopelvic examination (100.0) are highly predictive of the diagnosis. However, the yield of cases was very low (13\% for examination and 3.7\% for pain score). Bouguizane $e t$ al. concluded that clinicians must be aware of possible adnexal torsion in women with acute

Table 3. Association of the results of ultrasound and a definitive diagnosis of ovarian torsion with patient characteristics.

\begin{tabular}{lcc} 
Variable & Ultrasound & Diagnosis \\
Age & $0.61^{\mathrm{b}}$ & $0.12^{\mathrm{b}}$ \\
Marital Status & $0.068^{\mathrm{b}}$ & $0.71^{\mathrm{a}}$ \\
\hline Pregnant & $0.30^{\mathrm{b}}$ & $0.47^{\mathrm{b}}$ \\
Pain Score & $0.008 \mathrm{~b}^{*}$ & $1.00 \mathrm{a}$ \\
\hline Vomiting \& nausea & $0.90^{\mathrm{b}}$ & $0.42^{\mathrm{b}}$ \\
White blood cells & $0.32^{\mathrm{b}}$ & $0.47^{\mathrm{a}}$ \\
\hline Clinical examination & $0.039^{\mathrm{b} *}$ & $0.86^{\mathrm{b}}$ \\
Pathology & $0.43^{\mathrm{b}}$ & $0.22^{\mathrm{b}}$ \\
\hline
\end{tabular}

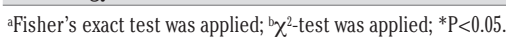


Table 4. Predictive value and yield of different criteria for the diagnosis of ovarian torsion.

\begin{tabular}{|c|c|c|c|c|c|c|}
\hline Criteria for diagnosis & Positive & Negative & Total & PPV (\%) & Yield (\%) & \\
\hline US only & 0 & 1 & 1 & 0.0 & 0.0 & \\
\hline Examination only & 7 & 0 & 7 & 100.0 & 13.0 & \\
\hline Pain score only & 2 & 0 & 2 & 100.0 & 3.7 & \\
\hline US + exam. & 14 & 3 & 17 & 82.4 & 25.9 & \\
\hline US + pain score & 6 & 1 & 7 & 85.7 & 11.1 & $74 \% *$ \\
\hline US + pain score + exam & 20 & 3 & 23 & 87.0 & 37.0 & \\
\hline Pain + exam. & 1 & 0 & 1 & 100.0 & 1.9 & \\
\hline All negatives & 4 & 0 & 4 & 100.0 & 7.4 & \\
\hline Total & 54 & 8 & 62 & & & \\
\hline
\end{tabular}

US, ultrasound; PPV, positive predictive value; *this figure reflects the yield of ultrasound in addition to examination and/or pain score.

pelvic pain; ${ }^{9}$ ultrasound is a useful tool in these situations. ${ }^{10}$ In the present study, the predictive value of US and pain score is $85.7 \%$, but the number of cases is very low (11.1\%).

Ignacioa et al. concluded that an ultrasound image can usually be used to make a diagnosis in conjunction with clinical parameters; ${ }^{25}$ however, this is most difficult in patients with ovarian torsion. In the present study, the predictive value of US with only examination is $82.4 \%$, but again, the yield is low (25.9\%). However, when relying upon US in conjunction with either examination and/or the pain score, the yield reached $74 \%$. These were the only two variables that had positive associations with ultrasound $(\mathrm{P}=0.008$ for the pain score and $\mathrm{P}=0.039$ for the examination). Clinical assessment by nausea-vomiting, pain score, age group, marital status, and pregnancy status was not statistically significant in predicting ovarian torsion, although the PPV was high for leukocytosis (0.92), existing ovarian pathology $(0.89)$, and nausea-vomiting $(0.87)$.

\section{Conclusions}

From the collective findings of this study, and considering its limitations in terms of sample size, it is concluded that the definitive diagnosis of ovarian torsion remains challenging. Both clinical and sonographical evaluation of acute pelvic pain should be considered for the diagnosis of ovarian torsion. The diagnosis cannot be exclusively based on ultrasound only, on the presence or absence of color flow Doppler, or even on the morphological findings. Therefore, surgical intervention is recommended in suspicions of a nonviable ovary in order to decrease the morbidity.

\section{References}

1. Houry D, Abbott JT. Ovarian torsion: a fifteen-year review. Ann Emerg Med
2001;38:156-9.

2. Becker JH, de Graff J, Vos CM. Torsion of the ovary: a known but frequently missed diagnosis. Eur J Emerg Med 2009;16:124-6.

3. Vaaras M, Tsikini A, Polyzos,D, et al. Uterine adnexal torsion: Pathologic and gray-scale ultrasonographic findings. Clin Exp Obstet Gynecol 2004;31:34-8.

4. Sommerville M, Grimes DA, Koonings PP, et al. Ovarian neoplasms and the risk of adnexal torsion. Am J Obstet Gynecol 1991; 164:577-8.

5. Ander, JF, Powell EC. Urgency of evaluation and outcome of acute ovarian torsion in Pediatric Patients. Arch Pediatr Adolesc Med 2005;159:532-5.

6. Mashiach R, Canis M, Jardon $\mathrm{K}$, et al. Adenexal torsion after laparoscopic hysterectomy: description of seven cases. J Am Assoc Gynecol Laparosc 2004;11:336-9.

7. Littman ED, Rydfors J, Milki AA. Exerciseinduced ovarian torsion in the cycle following gonadotrophin therapy: case report. Hum Reprod 2003;18:1641-2.

8. Beaunoyer M, Chapdelaine J, Bochard S, et al. Asynchronous bilateral ovarian torsion. J Pediatr Surg 2004;39:746-9.

9. Bouguizane $\mathrm{S}$, Bibi $\mathrm{H}$, Farhat $\mathrm{Y}$, et al. [Adnexal torsion: a report of 135 cases]. [Article in French]. J Gynecol Obstet Biol Reprod (Paris) 2003; 32:535-40.

10. Born C, Wirth S, Stabler A, et al. Diagnosis of adnexal torsion in the third trimester of pregnancy: a case report. Abdom Imaging 2004;29:123-7.

11. Gorkemli H, Camus M, Clasen K. Adnexal torsion after gonadotrophin ovulation induction for IVF or ICSI and its conservative treatment. Arch Gynecol Obstet 2002;267:4-6.

12. Yen CF, Lin SL, Murk W, et al. Risk analysis of torsion and malignancy for adnexal masses during pregnancy. Fertil Steril 2009;91:1895-902.

13. Bromley B, Benacerraf B. Adnexal masses during pregnancy: accuracy of sonographic diagnosis and outcome. J Ultrasound Med 1997;16:447-52.
14. Schmeler KM, Mayo-Smith WW, Peipert JF, et al. Adnexal masses in pregnancy: Surgery compared with observation. Obstet Gynecol 2005;105:1098-103.

15. Johnson TR Jr, Woodruff JD. Surgical emergencies of the uterine adnexae during pregnancy. Int $\mathbf{J}$ Gynecol Obstet 1986;24:331-5.

16. Bayer AI, Wiskind AK. Adnexal torsion: can the adnexa be saved? Am J Obstet Gnycol 1994;171:1506-10. discussion 1510-1.

17. Pinto AB, Ratt, VS, Williams DB, et al. Reduction of ovarian torsion 1 Week after embryo transfer in a patient with bilateral hyperstimulated ovaries. Fertil Steril 2001;76:403-6.

18. Cohen SB, Wttiez A, Stockheim D, et al. The accuracy of serum interleukin- 6 and tumour necrosis factor as markers for ovarian torsion. Hum Reprod 2001;16: 2195-7.

19. Servaes S, Zurakowski D, Laufer MR, et al. Sonographic findings of ovarian torsion in children. Pediatr Radiol 2007;37:446-51.

20. Ben-Ami M, Perlitz Y, Haddad S. The effectivness of spectral and color Doppler in predicting ovarian torsion. A prospective Study. Eur J Obstet Gynecol Reprod Biol 2002;104:64-6.

21. Lee EJ, Kwon Hc, Joo HJ, et al. Diagnosis of ovarian torsion with color Doppler sonography: depiction of twisted vascular pedicle. J Ultrasound Med 1998;17:83-9.

22. Hibbard LT. Adnexal torsion. Am J Obstet Gynecol 1985;152:456-61.

23. Pena JE, Ufberg D, Coony $\mathrm{N}$, et al. Usefulness of Doppler sonography in the diagnosis of ovarian torsion. Fertil Steril 2000;73:1047-50.

24. Cohen SB, Weisz B, Seidman DS, et al. Accuracy of the preoperative diagnosis in 100 emergency laparoscopies performed due to acute abdomen in non-pregnant women. J Am Assoc Gynecol Laparosc 2001;8:92-4.

25. Ignacio EA, Hill MC. Ultrasound of the acute female pelvis. Ultrasound $Q$. 2003;19:86-98; quiz 108-10. 